\title{
Points cardinaux pour la conception de formations universitaires pluridisciplinaires en épistémologie et histoire des sciences pour les enseignants du secondaire, ou comment s'appuyer sur des dilemmes
}

\author{
Alain Bernard ${ }^{1}$, Frédéric Brechenmacher ${ }^{2}$ et Matthieu Husson ${ }^{3}$ \\ ${ }^{1}$ Université Paris Est Créteil, ESPE de l'académie de Créteil (groupe « Sciences et techniques en \\ interférences »), Centre Koyré (CNRS, UMR8560), labex HASTEC (ANR-10-LABX-85), France \\ ${ }^{2}$ École polytechnique, LinX : Laboratoire interdisciplinaire de l'X - humanités et sciences sociales. \\ ANR CaaFÉ (ANR-10-JCJC 0101), France \\ ${ }^{3}$ CNRS-Observatoire de Paris, Laboratoire SYRTE (UMR 8630). ERC SAW (Grant 2010), France
}

\begin{abstract}
Résumé. Cet article s'intéresse à quelques uns des problèmes qui sous-tendent la conception des formations en épistémologie et histoire des sciences aujourd'hui offertes en France aux enseignants du second degré, en réponse à la demande croissante qui leur est faite. Ayant relevé plusieurs points communs incontournables à toute entreprise de formation universitaire s'adressant à ce type de public, notre objet principal est de proposer quelques points cardinaux, c'est-à-dire quelques principes d'orientation permettant de guider la conception de ce type de formation, pour autant qu'il entende concilier les exigences de pertinence, c'est-à-dire d'être adapté au public visé, et de rester ancré dans la recherche universitaire. Concernant le problème de la pertinence des formations, nous proposons préalablement deux points de vue complémentaires pour cerner ces contraintes: (1) le premier s'intéresse aux structures institutionnelles ainsi qu'aux sources disponibles qui conditionnent le travail des enseignants ; (2) le second se concentre sur l'activité des enseignants et résume les principaux dilemmes professionnels qu'ils doivent affronter alors qu'ils investissent au cœur de leur enseignement un champ interdisciplinaire comme l'est l'histoire et l'épistémologie des sciences. (3) Ce double point de vue nous permet alors de proposer un ensemble de points cardinaux pour la conception de formations universitaires permettant aux enseignants ou futurs enseignants de développer leur pouvoir d'agir dans ce domaine.
\end{abstract}

\section{Introduction}

Cet article s'intéresse à quelques uns des problèmes qui sous-tendent la conception des formations en épistémologie et histoire des sciences aujourd'hui offertes en France aux enseignants du second degré, en réponse à la demande croissante qui leur est faite. Aux enseignants de sciences mathématiques et expérimentales, on demande en effet, depuis maintenant plusieurs décennies, d'inclure une perspective 
culturelle, philosophique et historique dans leur enseignement. ${ }^{1}$ Des enseignants d'histoire, on attend qu'ils intègrent davantage l'histoire des sciences et des techniques à l'histoire générale, notamment sur toutes les parties relatives aux époques modernes et contemporaines $\left(16^{\text {ème }}-20^{\text {ème }}\right.$ siècles). En général, ces demandes s'inscrivent désormais dans un système prescriptif en mutation, qui laisse une large place à des orientations éducatives en lien à des questions sociétales et, par rebond, une place accrue à une perspective historique et épistémologique dans l'enseignement intégré des sciences.

Les formations universitaires touchant directement ou indirectement l'épistémologie et histoire des sciences et des techniques (EHST) présentent une grande diversité de pratiques. ${ }^{2}$ Elle doit l'être, pour au moins deux raisons simples. La première est que ce champ de recherche et d'enseignement reste, en France comme à l'étranger, d'une extension modeste quant à ses spécialistes stricto sensu. ${ }^{3}$ La seconde est qu'il est par nature caractérisé par une diffusion floue dans le vaste ensemble des enseignements universitaires pour lequel ce champ a une pertinence. En effet, de nombreuses disciplines universitaires contribuent d'une part à la formation des enseignants, et intègrent de l'autre une perspective épistémologique et/ou historique sur les sciences et les techniques. On peut citer, a minima, l'histoire, la philosophie, la sociologie ou l'anthropologie des sociétés anciennes et contemporaines, les sciences dites «dures » et la technologie, leurs didactiques, et les sciences de l'éducation. Pour toutes ces disciplines, l'EHST apparaît comme un «bien commun » et son identité est pour ainsi dire « diffractée » par les problématiques propres à chacune.

On doit donc s'attendre à une grande diversité d'approches et de points de vue sur ce type de formation, ainsi qu'à un degré d'expertise varié des formateurs. En effet, certains enseignants ont fait de l'EHST leur domaine de recherche, tandis que d'autres en ont une connaissance éclairée, le plus souvent intégré aux perspectives d'un domaine de recherche différent. Ce constat revient à dire que les problématiques de l'enseignement de l'EHST s'intègrent plus généralement à celles de l'enseignement des sciences humaines et sociales (SHS), qui faisaient le sujet du colloque UPEC de $2013 .{ }^{4}$ Les circonstances favorables à ce type de formations, souvent souhaitées mais peu soutenues dans les faits, sont changeantes et obligent les enseignants formateurs à des stratégies d'adaptation constantes.

L'objet de cet article n'est donc en aucune manière de proposer une quelconque normalisation de ces pratiques : au regard des constats rappelés ci-dessus, cette ambition serait absurde autant que contreproductive, la variété soulignée ci-dessus étant évidemment une richesse. Par contre, nous soutenons qu'on peut relever plusieurs points communs incontournables à toute entreprise de formation qui tâche de réponde à la demande soulignée ci-dessus : (a) elles prennent en charge la formation d'enseignants ou de futurs enseignants du secondaire, donc d'un système en partie compartimenté en disciplines distinctes et qui sont par conséquent travaillées de l'intérieur par des tensions institutionnelles qui ont des racines profondes et anciennes ; (b) elle gagne à prendre en compte les dilemmes professionnels vécus par les enseignants, à chaque fois qu'ils doivent s'emparer de l'EHST non pas seulement comme d'un simple objet d'intérêt culturel, mais comme d'un élément possible de leurs pratiques professionnelles ; enfin (c) elle privilégie par définition des savoirs, méthodes et approches universitaires, donc liés à des domaines et des méthodes de recherche.

A partir de ces constats qui nous semblent consensuels, notre objet principal est de proposer quelques points cardinaux, c'est-à-dire quelques principes d'orientation permettant de guider la conception de ce

\footnotetext{
${ }^{1}$ Cette attente est prégnante depuis les nouveaux programmes de 1993 au moins, lesquels tiraient déjà les conséquences d'expérimentations issus des grandes réformes des années 70. Voir (Fauque 2006, partie IV).

${ }^{2}$ En France comme en Europe, c'est ce dont témoignent les sessions spécifiquement dédiées à l'enseignement de l'histoire des sciences et des techniques dans les congrès français ou européens d'histoire des sciences.

3 Autrement dit le nombre d'enseignants qui investissent directement ou directement ce champ en formation, dépasse de loin celui des spécialistes. Voir à ce sujet (Lecourt 2000, 31-36).

${ }^{4} \mathrm{La}$ symposium consacré à l'enseignement, du dernier congrès de la Société Française d'Histoire des Sciences et des Techniques (SFHST) portait précisément sur cette question. Voir l'argumentaire détaillé sur le site du colloque http://sfhst2014lyon.sciencesconf.org/resource/page/id/5 (consulté 17.9.14).
} 
type de formation, pour autant qu'il entende concilier les exigences de pertinence (points a et b cidessus), c'est-à-dire d'être adaptée à son public et d'ancrage dans la recherche universitaire (point c). A contrario, il ne s'agit ni de proposer une norme, ni même de désigner des "passages obligés » pour ce type de formation; il s'agit plus modestement de proposer des principes d'orientation, des points cardinaux qui ne définissent aucun chemin particulier mais fournissent néanmoins une sorte d'orientation globale permettant d'éviter écueils et naufrages.

Les bases de notre discussion sont à la fois théoriques et pratiques. Nos cadres théoriques sont empruntés tout d'abord à l'histoire des disciplines scolaires, à titre de champ de recherche ainsi que d'objet d'enseignement possible. Il existe de manière plus générale une littérature de recherche qui s'est intéressée, au moins du côté des sciences et techniques (mathématiques, physique, biologie et technologie) aux questions d'intégration d'une perspective historique et philosophiques dans les enseignements correspondants. ${ }^{5}$ Cette littérature souffre elle-même du caractère souvent très marqué disciplinairement de la réflexion, alors qu'un nombre important des problèmes posés ne peuvent se comprendre dans le seul contexte de disciplines particulières d'enseignement $;^{6}$ en outre elle a longtemps eu (et souvent, garde) un caractère apologétique et militant qui tend à oblitérer les difficultés, en réalité importantes, qui font obstacle, pour beaucoup d'enseignants « non militants » à ce type d'entreprise pédagogique. $^{7}$ Finalement ça n'est qu'assez récemment, et de manière corrélative à l'inscription institutionnelle de la demande d'histoire dans les curricula de sciences, qu'on s'est intéressé aux difficultés propres à ce type d'enseignement qui intègre une double perspective, scientifique et historique et qu'on a proposé des solutions pratiques ayant fait l'objet d'expérimentations. ${ }^{8}$ C'est donc à cette perspective critique que nous entendons ici contribuer.

A ces champs relativement ciblés sur l'objet même des formations qui nous intéressent ici, nous ajoutons les didactiques disciplinaires, et plus particulièrement ses développements les plus récents qui font appel aux théories de l'activité et des communautés de pratique (Gueudet, Pepin, and Trouche 2011). Nous puisons également dans les champs de sociologie de l'éducation qui ont développé, en général, une approche des pratiques enseignantes contemporaines par l'examen des « dilemmes professionnels » que les enseignants doivent affronter au quotidien, en lien à la complexification récente de leurs tâches (voir par exemple Tardif, Lessard, and Dubet 1999). Nous empruntons également aux approches cliniques de l'activité professionnelles des enseignants (Pagoni 2010), tout particulièrement l'approche développée en psychologie du travail et qui donnent une place centrale à la gestion raisonnée et collective de dilemmes professionnels vécus en situation, gestion réflexive dont l'objectif est de favoriser le développement du pouvoir d'agir des acteurs. (Prot et al. 2010 ; Clot 2008 ; Roger 2007) : c'est en effet, selon nous, ce type « d'introspection professionnelle » qui se trouve au cœur de l'activité concrète des enseignants lorsqu'ils doivent incorporer une perspective historique à leur enseignement.

D'un point de vue pratique, nous n'avons aucune prétention ici à proposer des donnes nouvelles collectées suivant une méthodologie dédiée. Nous nous fondons plutôt sur une série d'expériences

\footnotetext{
${ }^{5}$ Pour l'enseignement des mathématiques, ces recherches sont bien représentées et suivies par le groupe HPM (History and Pedagogy of Mathematics) et une étude ICMI a déjà paru sur le sujet (Fauvel and Maanen 2000); pour les rapports entre l'histoire des sciences et l'éducation scientifique en général, le groupe IHPST, associé à la publication « science and education » publie régulièrement des synthèses sur la question (Matthews 2013).

${ }^{6}$ Du point de vue des contenus enseignés eux-mêmes, il est généralement reconnu que l'histoire des sciences est précisément un des vecteurs qui permettent de comprendre l'unité des questions ou des problèmes qui, tardivement, ont donné lieu à des traitements séparés.

${ }^{7}$ Il est constitutif de ce type d'enseignement de ne pouvoir être réduit à une simple question d'apprentissage dont il serait possible d'isoler les paramètres en dehors de toute considération sur la culture propre à l'enseignant (Barbin et al. 2002). Au contraire, cette liaison intrinsèque entre la formation des enseignants, celles des élèves et le partage d'une culture commune qui dépasse les deux, pose des problèmes d'approche délicats.

${ }^{8}$ Pour un point de vue sceptique, voir par exemple (Fried 2001). Pour un exemple de réflexions à la fois théoriques et pratiques sur l'usage de sources historiques dans l'enseignement des mathématiques, voir (Jahnke 2000).
} 
variées qui soutient implicitement notre analyse, et nous fournira le moment venu des illustrations concrètes prolongeant notre analyse générale.

Nous procéderons en trois étapes : les deux premières correspondent aux hypothèses (a) et (b) cidessus et proposent donc une définition globale du jeu de contraintes qu'une formation en histoire et épistémologie doit, dans tous les cas, prendre en compte. Nous proposons deux points de vue complémentaires pour cerner ces contraintes, le premier s'intéresse aux structures institutionnelles ainsi qu' aux sources disponibles qui conditionnent le travail des enseignants (partie 1); le second se concentre sur l'activité des enseignants et résume les principaux dilemmes professionnels qu'ils doivent affronter alors qu'ils investissent au cœur de leur enseignement un champ interdisciplinaire comme l'est l'histoire et l'épistémologie des sciences (partie 2). Ce double préalable nous permettra alors de proposer, comme nous l'avons signalé plus haut, un ensemble de points cardinaux pour la conception de formations universitaires permettant aux enseignants ou futurs enseignants de développer leur pouvoir d'agir dans ce domaine (partie 3). Nous verrons en conclusion que les perspectives proposées ne concernent pas seulement les formations elles-mêmes, mais aussi les ressources adaptées (imprimées et digitales) qu'on peut aujourd'hui leur associer.

\section{Les contraintes structurelles qui complexifient l'enseignement secondaire de l'épistémologie et l'histoire des sciences}

Dans cette partie nous revenons plus en détail sur l'histoire longue à laquelle nous avons fait allusion en introduction. Nous insistons sur une histoire d'un siècle et demie structurée par une tension permanente entre une tendance à la séparation en secteurs disciplinaires distincts, et une tendance à une vision plus intégrée de l'enseignement (§1.1). Sur la base de cet essai de synthèse historique, nous nous intéresserons ensuite, dans l'esprit des recherches récentes sur les ressources vives dans l'enseignement (Gueudet, Pepin, and Trouche 2011), aux contraintes qui définissent de manière générale la « marge de manœuvre » d'un ou d'une enseignant-e du système secondaire s'intéressant aujourd'hui à l'histoire et l'épistémologie des sciences. Nous insisterons sur la variété des dispositifs institutionnels qui lui sont offerts (1.2) ainsi que sur les sources variées du savoir qui leur est accessible (1.3). Nous conclurons en indiquant la conséquence à tirer de cette analyse pour l'étude des dilemmes qui seront étudiés ensuite (1.4).

\subsection{L'histoire longue d'une orientation floue}

En France comme dans d'autres pays ayant suivi la même évolution historique, la « demande d'histoire et d'épistémologie » faite aux enseignants du secondaire est l'héritage d'une histoire déjà longue de plus d'un siècle. Les études d'histoire de l'enseignement ont en effet montré que ce type de demande est né avec la bifurcation, qui s'est installée progressivement au cours du $19^{\text {ème }}$ siècle dans l'enseignement secondaire, entre les cursus d'humanités classiques et les cursus spécialisés sur les sciences, mathématiques et techniques (Hulin 2011, 15-27). Cette double demande reflète donc une tension déjà ancienne dans l'enseignement contemporain, qui est partagé entre la conservation « centripète » d'une visée généraliste et humaniste qui tend à unifier l'enseignement secondaire vers des buts communs, et une tendance « centrifuge » lié à l'institutionnalisation de disciplines distinctes, notamment au niveau du lycée.

Ce que montre encore cette histoire, est que très tôt des divergences considérables sont apparues sur la place exacte à donner à cet enseignement quand il était effectivement souhaité : c'est ce dont témoigne par exemple l'enquête lancée en 1931 dans la revue L'enseignement scientifique et qu'a analysée Nicole Hulin (Hulin 2005).

Sans vraiment résoudre le vieux problème de la séparation de fait des cursus disciplinaires, la demande persiste aujourd'hui d'un enseignement généraliste qui offre aux élèves une vision culturelle 
unifiée, permettant de situer notamment les sciences et techniques dans le cercle plus large de la culture légitime. Les tentatives les plus récentes faites pour clarifier les buts de l'enseignement primaire et de collège autour de l'acquisition de compétences communes, ${ }^{9}$ font toutefois perdurer les divisions anciennes entre une culture scientifique et techniques d'un côté, et une culture humaniste de l'autre.

Par ailleurs les contenus de l'enseignement scientifique et technique sont très nettement marqués, toujours dans les dernières décennies, par un mouvement lourd d'ouverture à marche forcée vers des questions socialement vives, c'est-à-dire intéressant la société dans son ensemble et ne renvoyant pas par ailleurs à des procédures didactiques standardisées ou marquées par une longue tradition scolaire. Les questions « d'éducation à » (à la santé, au développement durable, à l'usage courant des statistiques...) se sont très profondément installées dans les programmes et dans les nouveaux référentiels issus des réformes européennes des politiques éducatives (Pagoni and Tutiaux-Guillon 2012). Les enseignements d'EHST se trouvent ainsi pris dans un mouvement plus global, très ouvert sur des questions sociétales et conforme au concept de «formation tout au long de la vie », qui en oriente largement l'esprit - du moins l'esprit attendu.

Ces dispositifs sont par ailleurs relativement déconnectés, en France du moins, de la structure traditionnelle des enseignements disciplinaires. En général, le point qui parât essentiel ici est le caractère flou et ouvert des injonctions institutionnelles qui accompagnent ces changements : puisqu'il s'agit de matières qui n'ont pas une tradition longue d'enseignement, qui sont considérées au contraire comme propices à des apprentissages « a-didactiques » et conformes à ce que l'on rencontre ensuite dans la vie, on ne trouve pas non plus de directive très structurées pour leur enseignement, ou au moins leur intégration à l'enseignement, sinon sous la forme de dispositifs ouverts dont il sera question plus loin $(\S 1.2)$. Ce problème a des répercussions importantes sur la pratique comme nous le verrons ensuite (§2.3). En outre ce mouvement global est à son tour indissociable d'un changement préconisé dans les démarches et méthodes.

L'idée qu'une formation adéquate soit proposée aux enseignants, pour leur permettre de répondre concrètement à ces attentes, n'est guère moins ancienne que ces dernières : dès le 19 ème siècle, des tentatives concrètes, plus ou moins couronnées de succès, ont vu le jour pour proposer aux enseignants de sciences et techniques une formation historique et/ou philosophique. Mais l'enseignement universitaire ayant lui-même subi un fort mouvement de disciplinarisation, la tendance de fond est allée à la compartimentation des formations par discipline. Aussi des problèmes équivalents apparaissentils au niveau des cursus de formation d'enseignants, comme au niveau des recherches didactiques disciplinaires qui les ont accompagnées depuis les années 70. Là aussi cependant, l'apparition de courants de recherches transversaux, centrés autour de l'ergonomie et des théories de l'activité, et lourdement corrélé à la pénétration des technologies numériques dans l'enseignement et la formation, met en tension et questionne cet éclatement disciplinaire.

\subsection{La diversité critique des dispositifs scolaires et qui permettent d'enseigner I'histoire des sciences et des techniques}

Si le desideratum général pour un enseignement d'histoire et d'épistémologie des sciences est donc ancien, la conséquence visible de cette histoire longue et structurante est l'insistance, très inégale suivant les disciplines concernées, que mettent les programmes d'enseignement à suivre sur certains sujets une démarche historique, qui peut aller jusqu'à l'étude de textes fondateurs. Plus généralement, plusieurs

\footnotetext{
${ }^{9}$ Décret instituant le socle commun de connaissances et de compétences en 2006, suite à la loi « pour l'avenir de l'école » de 2005, réforme du lycée de 2010 à 2012.
} 
corps enseignants se sont appropriés ces questions, favorisant ainsi l'émergence d'une préoccupation pour l'insertion d'une perspective historique et épistémologique dans l'enseignement. ${ }^{10}$

En outre cette tendance longue et largement tributaire d'habitudes et de conceptions disciplinaires, a changé de nature dans les dernières décennies. Cela est du à l'émergence rapide, et lourdement soutenue par l'internationalisation des réflexions sur les curricula scolaires, d'une approche intégrée et décentralisée visant l'acquisition de compétences dont beaucoup sont transversales aux enseignements traditionnels : formation à la citoyenneté, formation à l'orientation, formation aux TICE, ou encore formation aux compétences de haut niveau comme la prise d'initiative. De nouveaux dispositifs sont ainsi apparus qui ménagent un espace pour les recherches personnelles et accompagnées, comprenant l'exploration d'une documentation ouverte et complexe. Des démarches, comme la démarche d'investigation, sont rapidement passées au rang de doxa et impliquent à leur tour le passage par une phase documentaire et critique. Tous ces dispositifs sont autant d'espaces offerts à des enseignements « non conventionnels » au regard des enseignements disciplinaires classiques, comme sont ceux d'histoire et d'épistémologie des sciences. Mais ces dispositifs se sont multipliés, sont plus ou moins en cohérence avec les structures classiques, et offrent en bonne partie l'image d'une mosaïque complexe aux enseignants comme à leurs élèves.

A ces contraintes d'ordre institutionnel, qu'expliquent largement une histoire bi-séculaire $(\$ 1.1)$, s'ajoute une contrainte d'ordre culturel et qui touche à la diversité structurelle des sources du savoir historique et épistémologique convoqué par les enseignants.

\subsection{La diversité critique des sources du savoir historique et épistémologique des enseignants sur les sciences et techniques}

Ces sources ne se réduisent pas, et de très loin, aux sources savantes qui sont aujourd'hui construites par la recherche dans ce domaine. Si on se restreint à ces dernières, elles reflètent naturellement une grande diversité de productions et de postures épistémologiques. Les productions qui relèvent stricto sensu de l'histoire des sciences et des techniques obéissent en général à des traditions historiographiques différentes, la différence étant premièrement due aux divisions disciplinaires traditionnelles encore très prégnantes. En outre l'enrichissement progressif, au cours des dernières décennies, d'une perspective épistémologique classique, relevant essentiellement de la philosophie, par l'histoire générale, l'anthropologie et la sociologie, la philologie et la linguistique, et en général par les sciences humaines, a eu pour conséquence une sorte d'explosion des postures épistémologiques et des points de vue sous lesquels les sciences et techniques pouvaient être envisagées : le terme de « sciences studies » (en France STS) résume souvent cette confluence de points de vue possibles sur l'objet visé, cette confluence dissimulant une grande diversité d'approches possibles (Braunstein 2008). Pour le monde enseignant, il faut encore ajouter la prolifération des différentes perspectives didactiques, dont l'origine est récente et qui se sont emparées de problématiques épistémologiques suivant un tempo et des préoccupations différentes de celles de l'histoire ou de la philosophie des sciences (Hosson and Schneeberger 2011). Dans certaines disciplines enseignées, comme les mathématiques, des groupes de recherche-action ont réuni depuis longtemps des chercheurs et des enseignants de tous niveaux et ont produit des ressources adaptées. ${ }^{11}$ La diversité de ces productions est finalement un reflet de la diversité des disciplines qui produisent ou exploitent en les «diffractant » les recherches en EHST (cf. introduction).

\footnotetext{
${ }^{10}$ Dans le cas français et pour les sciences physiques et chimiques, voir (Fauque 2006) ; pour les mathématiques (Barbin 2006, partie I).

11 (Barbin 2006). Voir aussi le site des IREM de la commission « histoire et épistémologie ». http://www.univ-irem.fr/ spip.php?rubrique160 (consulté 8.7.13).
} 
Mais ces productions qu'on peut qualifier de savantes sont loin de définir le périmètre des sources de savoir disponibles : il faut naturellement compter, au minimum, avec une gamme bien plus large de ressources disponibles et pertinentes. Les ouvrages et sites à forte diffusion, dont une partie seulement est cohérente avec la recherche universitaire, sont une ressource d'autant plus prisée qu'ils sont faciles d'accès et orientés vers le lecteur, donc déjà tourné vers un public d'apprenants. De nombreux enseignants isolés de tout contact universitaire ou de groupes de réflexion, mais fortement portés dans certains cas par des milieux associatifs, produisent des sites, des ouvrages dont la qualité varie du très médiocre à un très haut niveau d'information, et dont les contenus sont inévitablement marqués par les préoccupations professionnelles de leurs concepteurs. Il en va de même des productions issues de la hiérarchie scolaire, qui est en France assez souvent coupée du monde universitaire : ces institutions « endogènes » engendrent également une documentation abondante et dont la disponibilité trouve des relais puissants dans les grands sites institutionnels comme Eduscol en France, ou encore les sites de chaque académie scolaire. ${ }^{12}$ En général l'essor très puissant d'un patrimoine numérique a accentué cette extrême diversité.

Cette complexité évidente des sources du savoir dans ce domaine renvoie donc à des acteurs et des positions épistémologiques très divers. Elle doit en outre être redoublée par une caractéristique intrinsèque à toute littérature historique, qu'on pourrait appeler l'effet de patrimonialisation : en effet tout enseignant, comme aussi tout élève d'ailleurs, est porteur d'un savoir historique et épistémologique qu'on a pu parfois qualifier de spontané. ${ }^{13} \mathrm{Ce}$ savoir n'est bien sûr spontané qu'en apparence. Il est lui-même le produit d'une trajectoire personnelle ou collective complexe et d'un apprentissage nourri à des sources diverses et sur une temporalité longue. C'est ce qui explique notamment les différences de cultures entre différentes générations d'enseignants. ${ }^{14} \mathrm{La}$ « spontanéité » en question renvoie donc bien moins à un savoir naïf qu'à une trajectoire complexe dans le temps et qui implique généralement une forme de réflexivité. Cet effet patrimonial implique que les enseignants (comme leurs élèves) convoquent ultimement, dans leur appréhension de l'historicité des savoirs scientifiques et techniques, des ressources que nous qualifierons ici de «fantômes ». Ces ressources peuvent bien être jugées dépassées ou absentes au regard du patrimoine scientifique aujourd'hui disponible et légitime, elles ont au moins le mérite indubitable d'avoir fait l'objet d'une appropriation longue. Cette patrimonialisation sous forme de culture personnelle a donc un effet puissamment démultiplicateur de la diversité présente des ressources : elle ajoute la dimension du temps à la diversité des savoirs légitimés dans le présent. ${ }^{15}$

Cette situation intrinsèquement complexe et cette démultiplication « au carré ${ }^{16}$ des ressources disponibles en histoires des sciences et des techniques, explique que l'insertion d'une telle perspective dans l'enseignement secondaire ne peut être bien comprise dans les termes d'une transposition didactique. Le savoir concerné, c'est-à-dire celui qui est réellement disponible aux enseignants, est en effet un objet multiforme dont la présence est ancienne. Ce savoir est en large partie patrimonialisé, inégalement didactisé, n'est pas toujours le reflet de travaux de recherche, et renvoie à des types très divers de ressources documentaires.

\footnotetext{
12 Cette situation est à rapporter à la situation de monopole créée, en France, par le contrôle étatique de l'enseignement. Puisque c'est l'Etat qui met à disposition ces ressources, ces dernières n'ont pas besoin d'être situées, ni leurs auteurs.

13 (Brousseau 2008), le texte français est disponible sur le site de G. Brousseau. http://guy-brousseau.com/1463/ epistemologie-et-formation-des-professeurs-2006/ (consulté 8.7.13).

14 C'est précisément cette temporalité propre à la culture épistémologique de chacun, en plus du fait qu'elle est mal décrite comme un « phénomène naturel », qui oblige à nuancer le qualificatif de «spontané ».

15 On trouve dans la contribution de K. Gosztonyi dans ce même volume (Gosztonyi 2015), une réflexion très suggestive sur la possibilité d'exploiter un corpus ainsi "patrimonalisé" aux yeux d'un certain nombre d'enseignants. Il s'agit en l'occurrence un corpus hongrois touchant à l'enseignement et la vulgarisation des mathématiques selon une philosophie très spécifique. Dans on étude K. Gosztonyi appréhende ce corpus tant qu'objet de recherche, cette appréhension pouvant déboucher sur de nouvelles formations.

${ }^{16}$ Une fois par leur hyper-disponibilité dans l'espace numérique, une seconde fois par leur patrimonialisation.
} 


\subsection{Des contraintes structurelles à la mise en évidence de dilemmes}

Les paramètres structurels que nous venons de décrire ont en commun deux caractères. Le premier est qu'ils sont des facteurs de complexité : ils placent tous les enseignants, très fondamentalement, dans des situations obligées de choix dans un environnement structurellement complexe et flou dans bien des cas. Le second est qu'on peut les rapporter, en définitive, à la tension entre perspectives disciplinaires et perspective généraliste, transversale ou interdisciplinaire, dont il a été question en introduction, et que les réformes les plus récentes exacerbent en quelque sorte. On peut donc voir en effet dans les contraintes structurelles actuelles une sorte de «traduction contemporaine » de ces tensions déjà anciennes que l'analyse historique peut en partie restituer.

Or à partir du moment où on identifie des facteurs de complexification de l'environnement professionnel, on entre aussi dans le champ des recherches engagées depuis maintenant trois décennies sur les questions relatives aux dilemmes professionnels des enseignants dans la situation désormais complexe qui est la leur. ${ }^{17}$ L'étude fine de ces dilemmes et de leur construction endogène, sur laquelle nous reviendrons plus loin, part généralement de la reconnaissance d'un environnement professionnel dégradé ou du moins fragilisé par la complexification des tâches, l'apparition de demandes contradictoires et la parcellisation du travail. L'analyse historique ci-dessus tenant lieu d'une telle reconnaissance, il nous faut maintenant proposer une première approche des principaux dilemmes vécus par les enseignants, en relation aux attentes culturelles et institutionnelles dont il est question ici.

\section{Les dilemmes vécus par les enseignants lorsqu'ils s'intéressent à une intégration de l'EHST dans leur enseignement}

\subsection{La notion de dilemme professionnel et son intérêt}

Dans une acceptation ancienne et d'abord employée par les sociologues de l'enseignement, on peut parler de dilemme professionnel lorsque les acteurs vivent des contradictions au cœur de leur activité. ${ }^{18}$ Ces dilemmes peuvent être créés par des circonstances institutionnelles conflictuelles, ou par des oppositions entre la culture personnelle et intime de l'acteur avec son environnement institutionnel. Nous emploierons dans cette partie, l'adjectif « professionnel» dans ce sens lui conservant une signification relativement faible et contextuelle.

Cependant Luc Ria et ses collaborateurs (Ria et al. 2001) rappellent judicieusement que la notion de dilemme trouve son origine dans la tragédie et la rhétorique ancienne, et désigne une alternative, un choix dont les deux issues sont contradictoires et aussi insatisfaisantes l'une que l'autre. L'intérêt de ce rappel étymologique et historique tient dans l'ambigüité de la notion, qui se retrouve dans le réemploi qu'en font aujourd'hui les sociologues et psychologues du travail. En effet, la notion classique de dilemme désigne d'une part un conflit intérieur entre des exigences personnelles ou différentes circonstances extérieures inconciliables entre elles, et ce au cœur même d'une action; mais elle désigne aussi sa théâtralisation, son expression ou explicitation dans le cadre d'une expression, d'un mythe ou d'une référence codée et reconnue comme telle.

Les psychologues du travail sont ainsi conduits logiquement à distinguer le «dilemme de l'activité » qui renvoie à un conflit intérieur en lien à un problème rencontré dans l'activité professionnelle, et le « dilemme professionnel » qui part de l'explicitation des dilemmes d'activité par un travail prolongé de reprise et d'explicitation entre pairs, pour qu'il entre finalement dans la sphère d'un genre professionnel

\footnotetext{
17 On peut consulter l'article de L. Ria et de ses collaborateurs qui résume de manière synthétique les particularités de cette approche de l'activité enseignante (Ria et al. 2001).

18 Voir par exemple (Tardif, Lessard, and Dubet 1999).
} 
élaboré à plusieurs. Nous reviendrons dans un second temps (§3) sur ce sens plus subtil et prenant en considération la nécessité d'expliciter des conflits intérieurs, pour expliciter nos " points de repères ».

Pour l'heure et sans prétendre, comme nous l'avons signalé en introduction, nous appuyer sur l'analyse détaillée et systématique de témoignages nombreux, mais en partant plutôt de notre expérience de formation, nous tâcherons de restituer certaines de ces contradictions classiques quand elles sont liées à l'intégration interdisciplinaire de l'histoire et de l'épistémologie des sciences dans l'enseignement secondaire. Plus précisément, nous examinons trois types de dilemmes: ceux qui sont liés au sentiment d'illégitimité (2.2), ceux qui ressortent d'un problème de contrat didactique avec les élèves (2.3) enfin ceux qui sont liés à « l'organisation de l'étude » (2.4). Nous conclurons sur les perspectives offertes en termes de développement professionnel (2.5).

\subsection{Les dilemmes engendrés par l’illégitimité relative de ce type d'enseignement}

L'effet naturel de la démultiplication « au carré » des ressources disponibles pour un enseignement d'EHST (cf. §1.1) est de plonger d'emblée les enseignants dans un champ par nature pluridisciplinaire et 'culturel' qui ne correspond que partiellement (et différemment suivant les disciplines) à la culture professionnelle très spécifique dont ils sont aussi les porteurs. Cet écart est assez souvent accentué par les incohérences de la hiérarchie scolaire car il est souvent traduit par l'opposition caricaturale, mais pourtant prégnante, entre culture personnelle et culture professionnelle. Ce problème fondamental touche aussi bien les enseignants d'histoire qui doivent s'aventurer, par souci de cohérence, à explorer des contenus scientifiques que leur formation ne les a pas préparé à aborder, que des enseignants formés par et aux sciences et techniques, qui doivent s'aventurer sur un terrain historique ou philosophique qu'ils ne reconnaissent pas tout à fait comme le leur. Les dilemmes potentiellement engendrés par cet écart doivent être évidemment relativisés, au regard des changements institutionnels récents, dont certains donnent au contraire une pleine légitimité à une approche ouverte et transdisciplinaire de l'enseignement des sciences et techniques (\$1.2). Il serait naïf cependant de considérer que ces inflexions et désidératas suppriment à elles seules les dilemmes d'illégitimité. L'éthique professionnelle des enseignants du secondaire reste largement conditionnée par leur structuration en corps professoraux ${ }^{19}$ d'où ils tirent une large partie de leur identité, et les élèves eux-mêmes sont les premiers gardiens des divisions disciplinaires prégnantes, incarnées pour eux par les systèmes d'examen classiques.

Ce dilemme d'illégitimité se rattache à une partie centrale de l'activité enseignante et désormais bien reconnue par la recherche en didactique, à savoir que tout enseignant est en permanence dans un travail de re-conception des ressources de son enseignement : c'est ce qui permet de parler de ressources vives, suivant le vocabulaire proposé par Trouche et alii (Gueudet, Pepin, and Trouche 2011, ch.2). Pour le type d'enseignement qui nous intéresse ici, cela les conduit inévitablement à une retraduction permanente des contenus historiques à leur disposition. Cela les amène à explorer une partie du paysage épistémologique complexe décrit en $\$ 1.1$ en privilégiant, quand c'est possible, les formes adaptées et pré-adaptées pour un contexte pédagogique (notamment la littérature écrite et déjà réélaborée par des enseignants) ainsi que les formes patrimoniales les mieux connues. En construisant leur propre récit historique, en partie au moyen d'un travail documentaire adapté à la pratique pédagogique, ils se placent à la fois dans une situation naturelle (car ce travail est un des cœurs du métier) et non naturelle et illégitime, car il leur faut alors composer avec des savoirs vastes et ouverts $(\S 1.3)$, où ils ne se reconnaissent spontanément que peu de « légitimité savante ». Ils se retrouvent placés dans une posture inconfortable car médiocrement reconnue : celle de devoir produire sur un terrain où on ne leur reconnaît pas a priori de compétence à le faire.

19 Elle remonte au début du $20{ }^{\text {ème }}$ siècle, voir (Héry 2005). 


\subsection{Les dilemmes liés à des problèmes de contrat didactique avec les élèves}

On y a fait allusion plus haut, les élèves participent à leur manière, en raison de leur adhésion aux paradigmes fondamentaux qui structurent leur scolarité et leurs modes d'évaluation habituels, au dilemme d'illégitimité dont il vient d'être question. Le caractère interdisciplinaire et ouvert des activités à caractère épistémologique ou historique est en effet susceptible de remettre en cause le contrat didactique entre l'enseignant, les élèves et le savoir disciplinaire. Ce qui représente éventuellement un espace de liberté et d'ouverture pour l'enseignant, peut représenter un espace d'insécurité pour certains élèves, notamment ceux qui doivent rester au plus proche des gestes élémentaires de l'espace scolaire. Ceux là réagiront souvent par des questions du type : «Doit-on prendre des notes ? « Faut-il savoir ceci pour le contrôle ? ». De même, ces activités historiques et épistémologiques sont susceptibles de renverser les hiérarchies installées dans la classe, et certains élèves peuvent légitimement se sentir trahis par la perte des repères qui leur sont habituels. Ainsi, l'expérience montre que les lectures de sources historiques, primaires ou secondaires, nécessitent une grande expérience et un certain « doigté » pour être mises en pratique et devenir fructueuses. Elles sont d'autant plus délicates qu'elles rejoignent un problèmes d'organisation de l'étude dont il sera question plus loin (\$2.3) : il n'est le plus souvent pas possible dans un cours de sciences, par exemple, de détailler le contexte ou le corpus dans lequel telle source historique prend sens, c'est donc à l'enseignant que revient la tâche délicate d'institutionnaliser, dans un cadre contraint, une situation pédagogique permettant d'en rendre la lecture possible et fructueuse.

Notons que l'existence d'espaces ou de dispositifs scolaires plus adaptés à des activités de ce type, aussi bien du point de vue de leur légitimité que de leur faisabilité, ne fait pas disparaitre par miracle les problèmes ici soulevés. Au contraire, on a montré que la juxtaposition, sans effet de continuité, entre des activités valorisantes, et le cours 'normal' de la progression pédagogique principale, peut produire des effets dévastateurs sur des élèves qui ne comprennent pas le passage d'un mode pédagogique à l'autre (Bautier and Rayou 2013).

\subsection{Les dilemmes liés à l'organisation de l'étude}

Par organisation de l'étude, nous désignons ici l'ensemble des paramètres à la fois subtils et contraignants qu'un enseignant doit prendre en compte, sur la durée, quand il organise une progression didactique. ${ }^{20}$ Le premier dilemme que rencontre de ce point de vue un enseignant de sciences ou de mathématiques qui veut intégrer à son enseignement une perspective épistémologique et historique, est que les savoirs disponibles $(\$ 1.3)$, même ceux qui sont apparemment « médiés » pour l'enseignement, ne sont pas en général sous-tendus par une organisation correspondant à l'ensemble d'un cursus. Ce fait majeur est largement indépendant du fait que ces contenus apparaissent ou non dans les programmes officiels : car quand ils le font, c'est en général ponctuellement. De même, les manuels de sciences ou d'histoire qui intègrent de tels contenus se contentent souvent d'incises ponctuelles sans regarder à aucune organisation globale (Guedj, Laubé, and Savaton 2007, pp. 3-5). Aussi ces incises sont elles généralement distinguées du contenu disciplinaire tant du point de vue de la forme (encadrés, pages « curiosités » etc.) qu' au niveau des contenus historiques. Ces derniers sont le plus souvent déconnectés des activités proposées aux élèves dans la discipline enseignée (anecdotes, curiosités, lecture d'un texte primaire, notice biographique etc.).

Du même coup, il est possible de reprendre ici un constat maintes fois soulignés dans les recherches les plus lucides à ce sujet : l'intégration réelle d'EHST dans l'enseignement secondaire une perspective épistémologique ou historique dans un cours de sciences est souvent une affaire délicate et mal soutenue par l'organisation courante et concrète de l'étude et de ses contraintes. C'est probablement

\footnotetext{
${ }^{20}$ Pour une catégorisation plus fine de ces contraintes, voir (Ruthven 2012, 87-91).
} 
ce qui explique que des enseignants, même enthousiastes au départ, en viennent à abandonner ce type d'activité qui suppose des choix approfondis de sources, des temps d'expérimentation étendus, de forme sophistiquées de mises en récit qui ne s'inventent pas en un jour (Siu 2006). Au mieux, elles sont menées à bien par des enseignants talentueux ou par des communautés de praticiens, mieux à même de gérer les tensions induites par les contraintes habituelles de l'enseignement et ses normes prégnantes. La valorisation de ces expériences réussies masque, paradoxalement, les tensions sous-jacentes.

\subsection{Conclusion : un espace de développement professionnel}

En conclusion, les dilemmes professionnels que nous venons de relever, ainsi que l'espace de contraintes décrits plus haut $(\$ 1)$, ne signifient nullement que les enseignements ici visées soient impossibles ou voués à l'échec. Les expériences réussies et fécondes dans ce domaine, et elles sont nombreuses, attestent au contraire de leur intérêt et de leur faisabilité. Ces constats impliquent simplement que ce type d'enseignement, qu'il soit conçu pour lui-même ou intégré à un cursus existant (notamment disciplinaire), nécessite des constructions de sens complexes de la part des praticiens, seuls à même de réaliser des médiations avec les contraintes de l'enseignement qui passe par le dépassement concret des difficultés en question, pour qu'ils deviennent des dilemmes professionnels au sens fort souligné plus haut, c'est-à-dire intégrés au genre professionnel des enseignants du secondaire. Avant tout, ces médiations nécessitent donc des choix assumés et surtout une explicitation collective de ces choix. Installer les enseignants dans une position de concepteurs de leurs activités à base historique ou épistémologique, nécessite d'assumer le fait qu'ils ont leur propre travail documentaire à construire, en acceptant que la légitimé du récit historique qui en résulte ne croise pas forcément les critères de légitimité d'un récit historique ou philosophique à caractère savant.

Cette conclusion partielle nous conduit inévitablement à la question complémentaire de la précédente : s'il faut favoriser un travail de conception ou de co-conception par les enseignants euxmêmes, quels sont les principes qui doivent présider à un tel accompagnement ? Comment penser la conception de formations d'enseignants pertinentes, au sens qu'elles prennent en compte le jeu de contraintes ici reconnu, et qu'elles permettent l'élaboration de ces dilemmes professionnels ?

\section{Points cardinaux pour une formation pertinente des enseignants du secondaire en épistémologie et histoire des sciences et des techniques}

Si les enseignants du second degré sont reconnus comme acteurs et concepteurs à part entière de leur documentation et de leurs stratégies pédagogiques, à plus forte raison faut-il reconnaître aux enseignants universitaires en charge de leur formation en épistémologie et histoire des sciences, une liberté égale de conception et donc une grande variété de pratiques. Le problème posé ici, comme on l'a vu en introduction, est d'étudier les moyens d'adapter la formation à la situation qui est faite aux enseignants, et aux dilemmes qu'ils sont susceptibles d'éprouver.

Pour proposer des points de repère utiles à ce travail, nous reviendrons tout d'abord sur le risque qu'il y a à sur-privilégier dans ces formations la littérature savante en épistémologie et histoire des sciences et des techniques, sur toute autre forme de littérature historique (§3.1). Puis nous expliciterons le rôle de « levier » très particulier que joue, vis-à-vis des dilemmes de légitimité, le champ de l'histoire de l'enseignement des sciences et techniques (\$3.2). Enfin nous généraliserons ce cas particulier pour aborder le point névralgique que constitue le moyen de permettre aux enseignants d'élaborer collectivement leurs dilemmes professionnels signalés ci-dessus (§3.3).

\subsection{Premier point de repère : prévenir les écueils liés à la légitimité savante en EHST}

On a vu plus haut $(§ 1.3)$ non seulement que la littérature savante en EHST n’est qu'une petite partie du vaste continent des ressources disponibles à l'enseignant (que ces ressources soient présentement 
disponibles ou fantômes, c'est-à-dire intérieurement disponibles), mais encore qu'il ne s'agit pas nécessairement de la partie le plus pertinente pour le travail documentaire enseignant (\$2.2). Ce dilemme intrinsèque au travail documentaire des enseignants peut le cas échéant renforcer leur sentiment d'illégitimité et il est potentiellement aggravé si la formation ou l'enseignement souligne, comme il est naturel de le faire, la différence entre une connaissance validée (vérifiée, correctement contextualisée, etc.) et le vaste continent des demi-vérités ou des légendes historiques. On aboutit à un paradoxe classique, qui implique qu'un enseignement légitime d'un point de vue scientifique, soit par là-même facteur de blocage. Le blocage concerne le processus non moins naturel de mise en récit à visée pédagogique, ce qu'on pourrait appeler « l'affabulation pédagogique » (sans connotation péjorative) : la visée de ce dernier n'est pas toujours la vérité ou l'exactitude, mais bien souvent l'édification et la motivation. Pour reprendre un exemple classique, peu importe que Thalès ait visité ou non l'Egypte pour en mesurer les pyramides : il suffit que la chose soit racontée pour inciter à réfléchir, à partir d'une anecdote attrayante et dont les leçons sont plurielles, à la mesure indirecte des solides pyramidaux (Serres 1995).

Le point crucial, de ce point de vue, est que compte tenu des évolutions de l'historiographie, il n'est pas (plus) vraiment légitime de discréditer ce travail d'affabulation pédagogique : si en effet, dans la lignée de ce qu'on a pu appeler le tournant pratique et herméneutique de l'historiographie, on reconnaît aux acteurs de l'histoire - qu'il s'agisse de collectivités ou d'individus - une posture épistémologique et un point de vue sur leur propre histoire qui doit être compté parmi les « faits » historiques, alors le travail d'écriture de l'histoire lui-même, ainsi que la composition et recomposition permanente de la mémoire, font partie des faits historiques légitimes et valorisables en tant que tels. Pour filer l'exemple ci-dessus proposé, le problème historique n'est plus de savoir si Thalès est allé ou pas en Egypte, mais de comprendre quel écrivain était Plutarque, quand il vivait et quelles étaient ses raisons pour mettre ainsi en scène un philosophe grec légendaire face à un symbole du pouvoir Egyptien. Reprendre cette affabulation revient alors simplement à suivre consciemment une tradition et à réactiver un patrimoine littéraire, et d'un point de vue scientifique à enrichir la lecture d'un épisode donné par une pluralité de niveaux de lecture, en l'occurrence littéraires, philosophiques et anthropologiques. ${ }^{21}$

Ce paradoxe touche également les recherches en didactique fondées en partie sur les recherches en épistémologie et histoire des sciences, mais dont l'objectif est essentiellement prescriptif : elles visent à penser directement des ingénieries de formation plutôt qu'a mettre les enseignant en capacité de les produire. On peut s'attendre à ce que cette visée prescriptive entre en conflit avec des pratiques confirmées par l'expérience personnelle ${ }^{22}$. A contrario, les développements plus récents de la recherche didactique, reconnaissant aux enseignants leur plein rôle d'acteurs et de concepteur de leurs ressources, offrent un dépassement authentique du paradoxe, dont le fondement repose en dernier lieu sur le même tournant « pratique et herméneutique » souligné plus haut.

\subsection{Deuxième point de repère : donner les moyens d'élaborer les dilemmes d'illégitimité par des savoirs ou des champs de recherche cathartiques}

Cependant, que la pratique enseignante naturelle consistant à concevoir des ressources vives puisse entrer en conflit avec des formes " étroites » de légitimité savante en histoire, n'est qu'un des aspects des dilemmes d'illégitimité qu'affrontent les enseignants du secondaire lorsqu'ils doivent de fait étendre leur expertise au-delà du seuil assigné par leur spécialisation professorale. En effet, ce poids d'illégitimité provient à la fois de la structure de l'institution (qui reste pourtant guidé par un idéal de formation généraliste), d'une éthique et d'une identité professionnelle elles-mêmes médiées

\footnotetext{
21 Voir, au sujet, de Plutarque et de la distinction qu'il faisait lui-même entre histoire et biographie, la note synthétique publiée en ligne par M. Hannick sur le site Bibliotheca Classica Selecta (Hannick 2012).

22 Pour une discussion critique de ce point de vue, voir (Roger 2007, pp. 70-73).
} 
par des associations disciplinaires, comme des modèles prégnants de partage des rôles portés par les élèves eux-mêmes. Comment alors théâtraliser et mettre à distance ce poids d'illégitimité, pour en permettre l'élaboration consciente et favoriser l'appropriation collective de solutions? Comment en faire un dilemme professionnel ? Pour cela la racine de ces dilemmes doit d'abord être située et comprise, à condition toutefois de s'en donner les moyens.

Une façon traditionnelle d'envisager cette mise en situation, ce changement d'échelle ou de perspective à partir desquels le dilemme vécu devienne sur-vécu et visible aux yeux même des acteurs, est de l'installer dans une perspective socio-historique : c'est le moyen, traditionnel depuis Durkheim, d'envisager ce changement de point de vue. On remarquera que c'est très précisément sur ce type d'analyse « englobante » et préliminaire que repose en large partie notre analyse préliminaire ( $\$ 1$ et 2). C'est encore sur lui qu'on peut s'appuyer pour expliciter le champ de contraintes que constitue la crise permanente des humanités et qui est sans cesse rediscutée depuis un siècle et demi au moins. ${ }^{23}$ L'histoire et la sociologie de l'enseignement des sciences et techniques font donc partie de ces savoirs qui se distinguent tout particulièrement par leur pouvoir cathartique vis-à-vis des dilemmes d'illégitimité : ils permettent d'opérer le déplacement nécessaire, qui rend ces tensions visibles et situables sur un échiquier historique, institutionnel ou politique plus large. ${ }^{24}$ Ces savoirs, pour être traditionnels dans la formation des enseignants du secondaire, ne sont probablement plus les seuls : les travaux d'enquêtes comparatives au niveau international, associées ou non à une perspective de didactique comparée, la philosophie de l'enseignement, ou des champs nouveaux comme celui des humanités numériques (qui renégocient aujourd'hui la définition même de la perspective humaniste traditionnelle), peuvent probablement jouer ce rôle.

Une remarque s'impose : si ces savoirs académiques fournissent des clés intellectuelles pour situer les dilemmes d'illégitimité, leur simple exposé théorique n'est pas forcément le moyen le plus efficace pour déclencher une prise de conscience de ces tensions structurelles. A cet égard, le moyen le plus simple reste certainement d'ouvrir les formations proposées à des publics disciplinaires mixtes : le jeu des échanges entre enseignants de disciplines différentes, mais tous intéressés pour une raison ou une autre à la philosophie et/ou l'histoire des sciences et des techniques, créée généralement un terrain favorable. Parmi ces enseignants, il ne faut pas omettre de convoquer des documentalistes, ce qui est une façon très simple d'incarner le mouvement historique qui, depuis quatre décennies, a renforcé le questionnement autour du caractère généraliste de l'enseignement secondaire. Pour beaucoup d'enseignants des disciplines classiques, cette présence permet également de changer l'image appauvrie de simples bibliothécaires qu'ont trop souvent les documentalistes, dont la mission est souvent mal comprise.

Une seconde façon, complétant et généralisant la précédente, est de concevoir la formation, quand c'est possible, comme une véritable initiation à la recherche : les champs relevés plus haut, comme en témoigne l'expérience longue d'instituts comme l'ex-INRP en France, sont en effet précisément ceux qu'investissent volontiers les enseignants lorsqu'ils entreprennent, sans interrompre leur activité professionnelle, une formation à la recherche (master ou doctorat).

A titre de premier exemple de telles formations, mentionnons une formation professionnelle mise en place par l'un d'entre nous comme satellite d'un projet de recherche interdisciplinaire mis en place depuis 2011 autour d'une catégorie générique de textes historiques, intitulée " séries de problèmes ». ${ }^{25}$

\footnotetext{
23 (Durkheim 1990 ; Kambouchner 2013, ch9, p.156 et seq).

${ }^{24}$ Le terme cathartique est détourné d'Aristote, qui donne au théâtre tragique une dimension « purificatrice » de certaines émotions par le jeu de leur mise en scène explicite : elles sont jouées à distance. Pour un argument plus développé sur ce point, voir (Bernard 2013).

${ }^{25}$ Le projet a été mis en place au sein du labex HASTEC (Histoire et Anthropologie des Savoirs, Techniques et Croyances) en collaboration entre plusieurs laboratoires d'histoire ou d'anthropologie. Voir http://www.labex-hesam.eu/fr/13hastec-presentation (consulté 15.9.14) pour un descriptif du labex, et le carnet de recherche http://problemata. hypotheses.org/.
} 
La particularité de la formation associée ${ }^{26}$ est de s'adresser à un public mixte de stagiaires, des enseignants de différentes disciplines (histoire, mathématiques, français d'un côté) et d'étudiants en histoire ou anthropologie - ou les deux. ${ }^{27}$ Ce croisement de publics est corrélatif d'une option méthodologique, qui consiste à croiser volontairement, dans le cœur de la formation et le choix des textes comme de leur mode de lecture, les interrogations professionnelles « éveillées » par cette lecture, et les interrogations de recherche sur le corpus lui-même. ${ }^{28} \mathrm{Ce}$ croisement est d'autant plus intéressant dans ce cas, que bien des séries de problèmes ont été conçues dans une visée pédagogique, de sorte que certaines d'entre elles peuvent être vues à la fois comme un héritage réappropriable, et comme un document historique qu'il est possible de contextualiser, de mettre à distance. ${ }^{29}$

\subsection{Troisième point de repère : envisager la formation comme un champ de transformation de l'activité enseignante}

Les deux points cardinaux proposés (\$3.1 et 3.2) ont profondément un élément commun, qui mérite généralisation. Les dilemmes signalés ci-dessus $(\$ 2)$ ne peuvent être considérés comme provenant de malentendus ou de défauts d'organisation institutionnels ou éditoriaux : leur racine est dans le deux cas une réalité constitutive, intrinsèque à un certain état des savoirs d'un côté, et à un paradoxe des institutions d'enseignement secondaire, de l'autre. Prétendre dépasser ces contraintes ne peut donc signifier les abroger ou s'en affranchir : non qu'il s'agisse d'une réalité immuable, mais leur consistance et leur durée relative excède largement le champ d'action d'un enseignant en exercice, de sorte qu'il doit compter avec ces contraintes, pour tout ce qui le concerne, comme sur une constante. Le problème est plutôt de s'appuyer sur eux, de tourner autour d'eux en créant un espace de complexité qui rend ce mouvement ou ce déplacement possible.

C'est ici l'endroit où nous devons rejoindre une des conclusions provisoires de notre première approche des « dilemmes vécus » par les enseignants (\$2). Les difficultés, et tout particulièrement celles qui touchent à l'organisation de l'étude, trouvent forcément une solution innovante, car il faut combler un vide, s'adapter à un manque, un défaut de didactisation qui, simultanément, fait tout l'intérêt de ce champ. Autrement dit, les dilemmes touchant à l'organisation de l'étude, doivent aussi se rattacher aux contraintes qu'il n'est pas donné de résoudre par simple abrogation : il faut compter, le plus souvent, avec un défaut structurel des matériaux historiques quant à l'organisation de l'étude. Trop ouvert, trop peu normalisé, il ne peut être incorporé à une séquence d'enseignement qu'au prix d'un travail important et, le plus souvent, collectif.

Le problème posé ici à la conception d'une formation adaptée, est donc à nouveau d'étudier les conditions auxquelles la formation devient par elle-même un terrain de développement de cette activité d'innovation "normale », car réactive à une difficulté intrinsèque. Comme on l'a vu (§2.1), les psychologues de l'activité qui travaillent dans une tradition vygotskienne ont élaboré des stratégies sophistiquées pour permettre une prise de conscience progressive, d'abord individuelle (mais dialoguante) puis collective, des dilemmes d'activité, pour toutes sortes de professions dont la profession

\footnotetext{
${ }^{26}$ Les deux premières sessions sont décrites dans les deux billets suivants : http://problemata.hypotheses.org/225 (2013-14) et http://problemata.hypotheses .org/172 (2012-13).

27 Il existe en Ile de France un nombre important d'enseignants qui sont demandeurs de formation continue en histoire et épistémologie des sciences, et une partie d'entre eux est prête à s'inscrire dans une formation diplômante.

${ }^{28}$ Ce croisement a fait l'objet en avril 2014 d'une séance du séminaire « sciences et techniques en interférences », dont l'annonce est lisible en ligne (http://interferences.hypotheses.org/628 consulté le 17.9.14). La carte mentale associée à la présentation est également disponible en accès libre (http://www.mindmeister.com/fr/ 399721189/lier-recherche-et-formation-professionnelle-version-du-10-4-14-lien-vers-le-cr-de-la-sance consulté 17.9.14 - voir notamment la bulle « concevoir la formation »).

${ }^{29}$ Cette « duplicité » de l'objet rejoint les remarques éclairantes faites par C. Bonah et J. Danet au sujet du film documentaire comme objet ambivalent, sujet d'histoire et sujet d'une projection possible de l'activité professionnelle. Voir leur contribution (Bonah and Danet 2015), §2.
} 
enseignante (Clot 2008). Il s'agit là de procédures extrêmes, originellement adaptées à des situations de souffrance au travail qui vont généralement bien au-delà, par leur profondeur, des dilemmes dont il a été question plus haut. A fortiori, des procédures trop ambitieuses ne sont pas donc forcément recommandables, mais l'esprit doit certainement être le même : créer les conditions pour «bouger » autour de l'intimité d'un dilemme vécu, en créant progressivement les moyens pour qu'un espace de mouvement se créée.

A titre d'exemple, et pour ce qui nous occupe ici, on peut procéder à partir d'une discussion libre entre pairs, mais orientée explicitement vers l'explicitation des difficultés habituellement rencontrées dans des essais plus ou moins fructueux pour intégrer une perspective épistémologique ou historique dans son enseignement. Ce sont, de ce point de vue, souvent les échecs ou les frustrations éventuelles qui, une fois explicitées, permettront une première libération par rapport au poids très particulier de la doxa apologétique qui entoure ces tentatives. C'est de cette façon que nous avons procédé pour une formation interdisciplinaire à l'histoire des sciences et des techniques qui s'est tenue entre 2009 et 2011 dans l'académie de Versailles sous le titre «Atelier d'histoire des sciences ». Cette formation regroupait une petite vingtaine de stagiaires provenant de nombreuses disciplines (mathématiques, Physique-chimie, Sciences et vie de la Terre, histoire-géographie, français, langues vivantes).

Un élément particulièrement représentatif de l'approche que nous avons employé dans cette formation est le « débat de problématisation » qui ouvre la formation. Sous le prétexte de donner la parole à chaque stagiaire afin qu'il présente ses attentes vis-à-vis de la formation, nous les amenons progressivement à exprimer les conditions de leur pratique (effective ou projetée) de l'histoire des sciences dans leurs activités d'enseignant. Une fois identifié, les différents traits de ces pratiques sont analysés par le dialogue et autour des dilemmes que nous avons identifiés précédemment. Ce débat permet ensuite de présenter les différents ateliers proposés par la formation. Chaque atelier mettant les stagiaires en position d'agir autour d'un des dilemmes que le débat de problématisation aura permis d'exprimer. Ces ateliers peuvent être modulés en fonction de l'implication et des besoins concrets des stagiaires.

\section{En guise de conclusion : les problématiques de conception de formations ou de ressources}

Les principes généraux que nous avons ici proposés et justifiés ne portent pas précisément sur des ingénieries particulières de formation, elles-mêmes trop diverses pour être subsumées sous un même modèle. On a vu plutôt qu'ils fonctionnent comme des repères pour permettre la conception de formations pertinentes, adhérentes à la réalité effective et concrète d'enseignants en exercice. A défaut de pouvoir produire un modèle de telles formations, nous avons proposé quelques exemples de formations dont que nous continuons à élaborer.

L'intérêt d'un énoncé général est par ailleurs qu'il permet de poser le délicat problème de la conception de ressources pour l'enseignement de l'histoire des sciences. On a vu plus haut que les matériaux traditionnels et «proximaux » de l'enseignement, comme les manuels courants ou les ressources institutionnelles, reflétaient, plutôt qu' ils ne résolvaient, les dilemmes liés à l'organisation de l'étude. La question se pose donc évidemment, d'étudier la meilleure manière de proposer des ressources à la fois fiables scientifiquement et ouvertes à un travail créatif de conception. Des modèles d'éditions de sources historiques existent, qui incorporent de manière plus ou moins prononcée et étudiée, des dimensions propres à l'enseignement. A titre d'exemple, le projet sur les « séries de problèmes » mentionné plus haut, s'oriente désormais vers la production de ressources commentées, intégrant des « commentaires enseignants » dans la conception même de la ressource. ${ }^{30}$

30 Voir un descriptif dans le préprojet en cours d'élaboration : http: //problemata.hypotheses .org/316 (consulté 17.9.14). 
Ce type de travail doit selon nous s'inspirer des mêmes orientations, que celles proposées ici à l'ingénierie de formation. Du reste la distinction des deux types de conception, notamment en raison de la montée en force des outils de communication et de conception numériques, tend à s'estomper : au vu du développement déjà important des recherches sur la conception des ressources pour l'enseignement, on doit s'attendre à ce que les deux types d'ingénierie iront désormais de pair. ${ }^{31}$

\section{Références}

[1] Evelyne Barbin. 2006. « Apports de l'histoire des mathématiques et de l'histoire des sciences dans l'enseignement », Tréma 26. URL : http://trema.revues . org/64

[2] Barbin, Evelyne, Giorgio T. Bagni, Lucia Grugnetti, Manfred Kronfellner, Ewa Lakoma, and Marta Menghini. 2002. "Integrating History : Research Perspectives." In History in Mathematics Education, edited by John Fauvel and Jan Van Maanen, 63-90. New ICMI Study Series 6. Springer Netherlands. http://link. springer. com/chapter/10.1007/0-306-47220-1_3.

[3] Bautier, Élisabeth, and Patrick Rayou. 2013. Les inégalités d'apprentissage : programmes, pratiques et malentendus scolaires. [2e éd. revue et augmentée. 1 vols. Éducation et société. Paris : Presses universitaires de France.

[4] Bernard, Alain. 2013. "Former Les Futurs Enseignants À L'histoire Des Disciplinaires Scolaires. » In Épistémologie, Savoirs et Champs Disciplinaires : Questions D’apprentissage, by Brigitte Marin and Dan Savatovsky, 15-28. Professeur Aujourd'hui. CRETEIL : CRDP de l'académie de Créteil.

[5] Bonah, Christian, and Joël Danet. 2015. « MedFilm. Enseigner « Par et Pour La Recherche » Les SHS En Milieu Médical À L'aide Du Film «Utilitaire». EDP Sciences. Dans ce volume.

[6] Braunstein, Jean-François, ed. 2008. L'histoire des sciences : méthodes, styles et controverses. Translated by Vincent Guillin and Anna C. Zielinska. 1 vols. Textes clés. Paris : J. Vrin.

[7] Brousseau, Guy. 2008. "L'epistemologia scolastica spontanea e la cultura dei problemi matematici." La matematica e la sua didattica, no. $4: 621-55$. Version française en ligne (consulté 8.7.13) : http://guy-brousseau.com/1463/epistemologie-et-formationdes-professeurs-2006/

[8] Clot, Yves. 2008. Travail et pouvoir d'agir. 1 vols. Le travail humain. Paris : Presses universitaires de France.

[9] Durkheim, Émile. 1990. L'évolution pédagogique en France. 1 vols. Quadrige 109. Paris : Presses universitaires de France.

[10] Fauque, Danielle. 2006. « La « longue marche » d'un enseignement de l'histoire des sciences et des techniques. » Tréma, no. 26 (October) : 34-47. http://trema.revues . org/83

[11] Fauvel, John, and J. A. van Maanen, eds. 2000. History in Mathematics Education - An ICMI Study (NEW ICMI STUDIES SERIES Volume 6) (New ICMI Study Series). 1 edition. Springer.

[12] Fried, Michael N. 2001. "Can Mathematics Education and History of Mathematics Coexist » Science \& Education 10 (4) : 391-408. doi:10.1023/A:1011205014608.

[13] Gosztonyi, Katalin. 2015. «Quelques Réflexions Sur Les Possibilités de L'enseignement de L'histoire Des Mathématiques Dans La Formation Des Enseignants En Hongrie. » EDP Sciences. Dans ce volume.

[14] Guedj, Muriel, Sylvain Laubé, and Pierre Savaton. 2007. « Vers Une Didactique de L'histoire Des Sciences Eléments de Problématiques et de Méthodologie Pour Une Didactique de L'épistémologie et de L'histoire Des Sciences et Des Techniques (EHST). » In Actes Du Congrès

\footnotetext{
${ }^{31}$ Pour un exemple d'approche intégrée, voir de nouveau la contribution de C. Bonah et de J. Danet dans ce même volume (Bonah and Danet 2015).
} 
Les sciences humaines dans les parcours scientifiques et techniques professionnalisants

AREF 2007, 12p. Strasbourg. http://www.congresintaref.org/actes_pdf/AREF2007_ Muriel_GUEDJ_285.pdf

[15] Gueudet, Ghislaine, Birgit Pepin, and Luc Trouche. 2011. From Text to "Lived" Resources : Mathematics Curriculum Materials and Teacher Development. Dordrecht; New York : Springer.

[16] Hannick, Jean-Marie. 2012. "PLUTARQUE." http://bcs.fltr.ucl.ac.be/encyc-1/ plutarque.htm.

[17] Héry, Evelyne. 2005. «Les Professeurs de Lycée et L'application de La Réforme de 1902. Vers de Nouvelles Pratiques Pédagogiques? »In Lycées, Lycéens, Lycéennes. Deux Siècles D’histoire, by Philippe Savoie, Pierre Caspard, and Jean-Noël Luc, INRP, 257-67. Lyon.

[18] Hosson, Cécile (de), and Patricia Schneeberger. 2011. «Dossier ? : Didactique Des Sciences et Histoire Des Sciences. » Recherches En Didactique Des Sciences et Des Technologies (RDST) 3 : pp. 7-152. http://rdst.revues.org/363

[19] Hulin, Nicole. 2005. "Histoire Des Sciences et Enseignement Scientifique Au Lycée Sous La Troisième République / History of Science and Science Teaching in Secondary Schools under the Third Republic . » Revue D'histoire Des Sciences 58 (2) : 389-405. doi: 10.3406/rhs.2005.2254.

[20] Hulin, Nicole. 2011. Culture scientifique et humanisme : un siècle et demi d'engagement sur le rôle et la place des sciences. 1 vols. Histoire des sciences humaines. Paris : l'Harmattan.

[21] Jahnke, H. N., ed. 2000. "The Use of Original Sources in the Mathematics Classroom." In J. Fauvel and J. van Maanen (Eds). History in Mathematics Education : The ICMI Study., 291-328. Dordrecht : Kluwer Academic Publishers.

[22] Kambouchner, Denis. 2013. L'école, question philosophique. 1 vols. Histoire de la pensée. Paris : Fayard.

[23] Lecourt, Dominique. 2000. «L'enseignement de La Philosophie Des Sciences, Rapport Au Ministre de L'éducation Nationale, de La Recherche et de La Technologie. » http://media. education.gouv.fr/file/94/7/5947.pdf.

[24] Matthews, Michael R., ed. 2013. International Handbook of Research in History, Philosophy and Science Teaching. Springer.

[25] Pagoni, Maria. 2010. "Approches cliniques des apprentissages. » Recherche \& formation $\mathrm{n}^{\circ} 63$ (1) : 17-22. http://rechercheformation.revues.org/331

[26] Pagoni, Maria, and Nicole Tutiaux-Guillon. 2012. «Les éducations à. . . : nouvelles recherches, nouveaux questionnements ?» Spirale - Revue de Recherches en Éducation, no. 50 (October) : 3-10. http://spirale-edu-revue.fr/spip.php?article1114

[27] Prot, Bernard, Joëlle Mezza, Régis Ouvrier-Bonnaz, Emmanuelle Reille-Baudrin, and Pierre Vérillon. 2010. «Les dilemmes d'activité. » Recherche \& formation n63 (1) : 63-76. http://recherchef ormation.revues.org/233

[28] Ria, Luc, Jacques Saury, Carole Sève, and Marc Durand. 2001. « Les dilemmes des enseignants débutants : Etudes lors des premières expériences de classe en Education Physique. » Science et Motricité 42 : 47-58. http: //hal . archives-ouvertes.fr/hal-00803976

[29] Roger, Jean-Luc. 2007. Refaire son métier : essais de clinique de l'activité. Edited by Conservatoire national des arts et métiers and France. 1 vols. Clinique du travail. RamonvilleSaint-Agne : Éd. Érès.

[30] Ruthven, Kenneth. 2012. "Constituting Digital Tools and Materials as Classroom Resources : The Example of Dynamic Geometry." In From Text to "Lived" Resources, edited by Ghislaine Gueudet, Birgit Pepin, and Luc Trouche, 83-103. Mathematics Teacher Education 7. Springer Netherlands. http://link.springer.com/chapter/10.1007/978-94-007-1966-8_5.

[31] Serres, Michel. 1995. Les origines de la géométrie : tiers livre des fondations. Champs 331. Paris : Flammarion. 
[32] Siu, Man-Keung. 2006. "No, I Don't Use History of Mathematics in My Class. Why" In Proceedings of HPM2004 \& ESU4., 268-77. F. Furinghetti et al, Uppsala Universitet. http://hkumath.hku.hk/ mks/10thICMI-MKS.pdf

[33] Tardif, Maurice, Claude Lessard, and François Dubet. 1999. Le travail enseignant au quotidien: expérience, interactions humaines et dilemmes professionnels. Bruxelles, Belgique, Canada, France. 